\title{
Diacronie
}

Studi di Storia Contemporanea

$N^{\circ} 35,3 \mid 2018$

Gli strumenti di Clio

\section{Commento di Giovanni Gozzini a Un'età contro la storia. Saggio sulla rivoluzione del XXI secolo}

\section{Giovanni Gozzini}

\section{(2) OpenEdition}

\section{Journals}

\section{Edizione digitale}

URL: http://journals.openedition.org/diacronie/9086

DOI: 10.4000/diacronie.9086

ISSN: 2038-0925

\section{Editore}

Association culturelle Diacronie

\section{Notizia bibliografica digitale}

Giovanni Gozzini, «Commento di Giovanni Gozzini a Un'età contro la storia. Saggio sulla rivoluzione del XXI secolo », Diacronie [Online], №35, 3 | 2018, documento 10, Messo online il 29 septembre 2018, consultato il 21 avril 2019. URL : http://journals.openedition.org/diacronie/9086 ; DOI : 10.4000/ diacronie.9086 


\section{Diacronie}

Studi di Storia Contemporanea

\section{$35,3 / 2018$}

Gli strumenti di Clio: uomini, luoghi e teorie della storia dalla tradizione critica alla comunicazione digitale

\section{Commento di Giovanni Gozzini a Un'età contro la storia. Saggio sulla rivoluzione del XXI secolo}

\section{Giovanni GOZZINI}

Per citare questo articolo:

GOZZINI, Giovanni, «Commento di Giovanni Gozzini a Un'età contro la storia. Saggio sulla rivoluzione del XXI secolo», Diacronie. Studi di Storia Contemporanea : Gli strumenti di Clio: uomini, luoghi e teorie della storia dalla tradizione critica alla comunicazione digitale, 35, 3/2018, 29/09/2018,

URL: < http://www.studistorici.com/2018/09/29/gozzini_numero_35/ >

Diacronie Studi di Storia Contemporanea $\rightarrow$ http://www.diacronie.it Rivista storica online. Uscita trimestrale.

redazione.diacronie@hotmail.it

Comitato di direzione: Naor Ben-Yehoyada - João Fábio Bertonha - Christopher Denis-Delacour - Maximiliano Fuentes Codera Anders Granås Kjøstvedt - John Paul Newman - Deborah Paci - Niccolò Pianciola - Spyridon Ploumidis - Wilko Graf Von Hardenberg Comitato di redazione: Jacopo Bassi - Luca Bufarale - Gianluca Canè - Fausto Pietrancosta - Alessandro Salvador - Matteo Tomasoni Diritti: gli articoli di Diacronie. Studi di Storia Contemporanea sono pubblicati sotto licenza Creative Commons 3.0. Possono essere riprodotti e modificati a patto di indicare eventuali modifiche dei contenuti, di riconoscere la paternità dell'opera e di condividerla allo stesso modo. La citazione di estratti è comunque sempre autorizzata, nei limiti previsti dalla legge. 


\title{
10/ Commento di Giovanni Gozzini a Un'età contro la storia. Saggio sulla rivoluzione del XXI secolo
}

\author{
Giovanni GOZZINI
}

Giovanni Gozzini nel suo commento al saggio di Giuseppe Carlo Marino su globalizzazione e crisi del "pensare storico" inquadra i cambiamenti nelle dinamiche economiche e sociali dell'ultimo trentennio in un più ampio mutamento del rapporto fra l'uomo e la realtà globalizzata del nuovo secolo. Gli eventi storici ricordati e i cambiamenti avvenuti sono organicamente relazionati così con le recenti evoluzioni del paradigma dello sviluppo economico e del dibattito culturale dei nostri giorni.

Il pamphlet di Marino ha un enorme merito, dal mio punto di vista: quello di puntare a una ricomposizione dei saperi storici in un tempo che è invece, al contrario, di frammentazione crescente. Cerco di spiegarmi. A me pare che la ricerca storica, non solo in Italia, stia vivendo una fase di specializzazione esasperata che produce notevoli approfondimenti su singoli oggetti di studio ma fatica a ritrovare visioni d'assieme. Abbiamo tanti case-study su realtà locali nel periodo fascista, su articolazioni organizzative del regime, su aspetti particolari delle sue politiche nei diversi campi. Ma non abbiamo un dibattito sui tratti di fondo della dittatura simile a quello che negli anni ottanta (e forse anche nel decennio successivo) accompagnò l'opera di Renzo De Felice ${ }^{1}$. Incidono su questa separazione diversi fattori - come dire? - strutturali: la scomparsa di scuole storiografiche riconoscibili, a sua volta frutto della contrazione delle risorse economiche a disposizione dell'accademia, l'impianto di un sistema di valutazione della ricerca - comunque enorme passo in avanti rispetto al passato, in termini di internazionalizzazione e contenimento della discrezionalità baronale - che afferisce ai singoli specialisti di settore e quindi implicitamente scoraggia i tentativi interdisciplinari, una appropriazione abusiva della storia da parte dei media secondo logiche estranee alla conoscenza scientifica, l'annullamento di un nesso

${ }^{1}$ Si vedano ANGELO, Giuseppe, Renzo De Felice Bibliografia (1953-2002), Salerno, Edizioni del Paguro, 2002; GENTILE, Emilio, Renzo De Felice. Lo storico e il personaggio, Roma-Bari, Laterza, 2003; AGA ROSSI, Elena, Fascismo e antifascismo nell'opera di Renzo De Felice, in GOGLIA, Luigi, MORO, Renato, FIORENTINO, Fiorenza (a cura di), Renzo De Felice. Studi e testimonianze, Roma, Edizioni di Storia e Letteratura, 2002, pp. 121-137, p. 121. 
forte tra storia e politica nel senso di una fondazione e legittimazione dei partiti nel passato della nazione in quanto strumenti di rappresentanza di interessi e settori della società civile.

Ben venga quindi lo sforzo che Marino mette in campo per tornare a pensare in grande. $\mathrm{E}$ grande lo riferisco sia agli orizzonti spazio-temporali del campo d'analisi, sia alle ambizioni euristiche (ma direi anche etiche) della sua ricerca. Proprio per questo vorrei concentrare il mio intervento su due punti critici, strettamente connessi tra loro: il concetto di mercato e il concetto di globalizzazione.

Mi pare che Marino colga il punto saliente della fase dell'età contemporanea che stiamo vivendo nella sottomissione di ogni settore della vita civile alla logica del mercato che piega i cittadini in consumatori ${ }^{2}$. È un dato che emerge con forza dalla esperienza quotidiana di ciascuno, ma che Marino riconduce all'egemonia di una ideologia specifica - il neoliberismo - che dal tempo di Reagan e Thatcher ha posto fine a una lunga fase storica di segno contrario, aperta dalla crisi del '29 e segnata dallo sviluppo del welfare state nelle sue declinazioni sia occidentali di stampo keynesiano, sia pianificatrici di stampo sovietico ${ }^{3}$. Vorrei però ricordare una piccola evidenza empirica di segno contrario. Nel dicembre 1978, qualche mese prima che il binomio su ricordato arrivi al potere nel mondo anglosassone, il congresso del partito comunista cinese lancia la parola d'ordine delle quattro modernizzazioni, la prima delle quali riguarda la liberalizzazione dei mercati rurali. I contadini reagiscono bene, nonostante le catastrofi (il Grande Balzo in Avanti della fine degli anni cinquanta, la Rivoluzione culturale di metà anni sessanta) che in passato hanno funestato le loro esistenze con diversi milioni di morti. Nel giro dei venti anni successivi quasi 400 milioni di loro, secondo i dati della Banca Mondiale, escono da una condizione di povertà e tutto il paese avvia una prepotente crescita economica che dura fino ad oggi. Tutto bene allora? No di certo, oggi la Cina ha un livello di ineguaglianza economica addirittura superiore a quello degli Stati Uniti: situazione paradossale per un paese che si definisce comunista ${ }^{4}$

Quello che vorrei però sottolineare è il diverso contesto storico e culturale nel quale la svolta «neoliberista» prende campo in Oriente e in Occidente. Nel primo è la presa d'atto, riferita anche all'esperienza dell'Unione Sovietica, che l'economia di stato non riesce a produrre benessere

${ }^{2}$ Cfr. MARINO, Giuseppe Carlo, Un'età contro la storia. Saggio sulla rivoluzione del XXI secolo, Palermo, Università degli Studi di Palermo, Dipartimento di Scienze Politiche e delle relazioni internazionali (DEMS), 2017.

${ }^{3}$ Si possono vedere a riguardo le riflessioni presenti nei contributi contenuti nel numero monografico di «Diacronie» curato da Luca Bufarale e Fausto Pietrancosa: Diacronie Studi di Storia contemporanea : Quando la classe operaia andava in paradiso. Le Sinistre europee nell' "età dell'oro" del capitalismo, URL:

< http://www.studistorici.com/dossier/n-9-gennaio-2012/ > [consultato il 17 luglio 2018]; PEDALINO, Antonio, Ascesa e declino del neoliberismo. Da Reagan e Thatcher ai giorni nostri, Roma, Aracne, 2017; BENVENUTO, Sergio, «Il liberismo ha fallito?», in Doppiozero, 18 ottobre 2017, URL:

< http://www.doppiozero.com/materiali/il-liberalismo-ha-fallito > [consultato il 17 luglio 2018].

${ }^{4}$ Cfr. HEMERLYK DONALD, Stephanie, BENEWICK, Robert, Atlante della Cina: capire la crescita economica più rapida del pianeta, Bologna, Il ponte, 2007; MARCHISIO, Oscar, Cina \& capitalismo, ovvero un matrimonio quasi riuscito, Roma, Sapere 2000; KOSHY, Yohann (tradotta in italiano da Andrea Fumagalli e Gabriele Battaglia), "Cina: un ordine post-neoliberista? Intervista a Martin Jacques», in Effimera Critica e sovversioni del presente», [originariamente pubblicata su The New Internationalist], URL: < http://effimera.org/cina-un-ordine-postneoliberista-intervista-martin-jacques-yohann-koshy/ > [consultato il 18 luglio 2018]. 
perché non riesce a determinare spinta imprenditoriale nelle persone. Incentivi ideologici e innovazioni tecnologiche non alzano la produttività perché si infrangono contro l'apatia e l'ostruzionismo dei soggetti che dovrebbero esserne protagonisti: qualche anno dopo Gorbacev si scontrerà con le medesime resistenze ${ }^{5}$. Per quanto riguarda l'Occidente, tra i prolegomeni del neoliberismo vorrei richiamare il libro oggi del tutto dimenticato di un marxista americano, James O'Connor, dal titolo La crisi fiscale dello stato ${ }^{6}$. Il libro esce nel 1969 e teorizza una prossima rivoluzione in Occidente determinata dal progressivo venir meno delle risorse a disposizione del welfare state e quindi dalla conseguente contrazione dei servizi sociali e relativa esplosione di malcontento. La previsione è sbagliata ma la diagnosi no. Solo a costo di un crescente indebitamento e di una parallela finanziarizzazione delle economie nazionali sarà possibile mantenere le prestazioni sanitarie, scolastiche, assicurative erogate dagli stati moderni. Reagan e Thatcher nascono di qui e la contraddizione analizzata da O'Connor rimane ancor oggi insoluta. La costruzione di una alternativa al neoliberismo non avviene perché si limita ad agitare valori astratti - direbbe Marx - per quanto ineccepibili come solidarietà e uguaglianza, invece di capire quali forze produttive liberare o mettere in moto per far tornare i conti statali all'altezza delle necessità sociali. Debito e finanza, quando esagerano, determinano storture drammatiche per quasi tutti (non per i super-ricchi che ne sanno approfittare).

Non ho citato a caso Marx e le forze produttive. Perché credo che lì si debba tornare. Per Marx il mercato è una condizione naturale della vita umana: esiste anche nel tempo della Chiesa che Marino riprende da Le Goff?. Anche prima della comparsa dei mercanti, gli esseri umani che rimangono fuori dei conventi si scambiano i beni che producono e al mercato affidano la propria sopravvivenza. La loro storia gradualmente libera quel mercato dalle coercizioni extraeconomiche di servitù e lignaggio per poi reintrodurne altre, in età contemporanea, dovute alla forza organizzata dei lavoratori dipendenti. Il libero mercato, razionale ed efficiente, è sempre stato un'astrazione teorica ma nel mercato spurio, irrazionale e poco efficiente, gli uomini e le donne sono costretti a vivere. Deng Xiaoping e Gorbacev hanno dovuto prendere atto che un mercato controllato dallo stato non funziona (Marx infatti non ne ha mai parlato) perché le forze produttive non si sviluppano a partire da un centro onnisciente, bensì dal brulichio delle iniziative che nascono dal basso con l'obbiettivo individuale o collettivo di migliorare la propria esistenza.

Mi stupisce, per fare un altro esempio, che il dibattito sui robot si appunti pressoché esclusivamente sulla loro natura labor-saving, di risparmio di posti di lavoro umani, anziché sullo

\footnotetext{
${ }^{5}$ Si veda ad esempio GORBACHEV, Mikhail, MLYNAR Zdenek, Conversations with Gorbachev: on Perestroika, the Prague spring, and the crossroads of socialism, New York, Columbia University Press, 2002; RUDE, Gerd, Enigma Gorbaciov, Milano, SugarCo, 1991; GALEOTTI, Mark, Gorbachev and his revolution, Basingstoke-London-New York, Macmillan - St. Martin's Press, 1997.

${ }^{6}$ O'CONNOR, James, La crisi fiscale dello stato, Torino, Einaudi, 1979.

${ }^{7}$ Cfr. LE GOFF, Jacques, Nel Medioevo: tempo della Chiesa e tempo del mercante, in ID., Tempo della Chiesa e tempo del mercante e altri saggi sul lavoro e la cultura nel Medioevo, Torino, Einaudi, 1977, pp. 3-23.
} 
sviluppo delle forze produttive cioè sulla capacità dei robot, intanto, di rendere meno alienato il lavoro di fabbrica. Per un marxista questa sarebbe la prima domanda da porsi. Ma anche dopo. Nella banca dati che con Tommaso Detti abbiamo utilizzato in L'età del disordine, raccolta con fatica dalle serie annuali dell'International Labor Office $^{8}$, emergono tendenze significative. Gli addetti al settore secondario (manifatture, miniere, energia) crescono di numero in tutto il mondo a un ritmo superiore a quello dell'incremento demografico, passando tra 1950 e 2010 dal 7\% al 9\% della popolazione mondiale. Ma i paesi sviluppati (nord America, Europa, Giappone) passano in percentuale sul totale mondiale da due terzi a un quarto. Viceversa l'aumento degli addetti al settore terziario negli stessi paesi avanzati sopravanza di gran lunga la perdita di posti di lavoro in industria e agricoltura. Quasi ovunque, ma non in Italia e in altri paesi dell'Europa occidentale: perché? I motivi sono diversi ma hanno tutti a che fare con la contraddizione tra forze produttive e rapporti di produzione: basso capitale umano cioè deficit di formazione finalizzata alle competenze necessarie per le nuove professioni del terziario avanzato (soprattutto nel campo delle nuove tecnologie informatiche della rivoluzione di cui parla Marino), ruolo autoreferenziale della pubblica amministrazione invece che di servizio alla popolazione, distrazione (altrettanto autoreferenziale) dei capitali finanziari impiegati dalle banche in debito e speculazione anziché prestito e investimento in nuove società di servizi. L'elenco potrebbe continuare e costituisce la base per una alternativa non illuministica al neoliberismo, che stando dentro al mercato costruisca la liberazione delle energie positive che stanno dentro agli esseri umani ${ }^{9}$.

Il secondo punto critico riguarda la globalizzazione. Intesa nella sua accezione più semplice movimenti internazionali di persone, merci, capitali, idee e informazioni - esprime un'altra condizione naturale dell'umanità. La genetica storica dimostra al di là di ogni dubbio che siamo tutti discendenti di uno stesso ceppo di DNA nato nel cuore dell'Africa centinaia di migliaia di anni fa e poi diffusosi in tutto il mondo grazie alle migrazioni. Le tre scoperte che Francis Bacon pone alla base della civiltà moderna (polvere da sparo, bussola, stampa) arrivano in Europa dalla Cina portate dalle carovane arabe tra X e XIII secolo. Le ricerche dei fratelli Lucassen, tra le molte che si possono citare, restituiscono un quadro delle società europee in epoca moderna molto più mobile di quanto finora si sia pensato, con tassi significativi di migrazioni interne composte da soldati, marinai, mercanti, lavoratori stagionali. Una nave da trasporto risalente al 1300 a.C.,

\footnotetext{
${ }^{8}$ DETTI, Tommaso, GOZZINI, Giovanni, L'età del disordine: storia del mondo attuale 1968-2017, Roma-Bari, Laterza, 2018.

${ }^{9}$ Si vedano a riguardo le considerazioni contenute in GALLINO, Luciano, Globalizzazione e disuguaglianze, Roma-Bari, Laterza, 2000; ID., Il costo umano della flessibilità, Roma-Bari, Laterza, 2001; ID., Il lavoro non è una merce. Contro la flessibilità, Roma-Bari, Laterza, 2007; ID., Finanzcapitalismo. La civiltà del denaro in crisi, Torino, Einaudi, 2011.
} 
recentemente riportata alla luce al largo delle coste turche, ha svelato un carico contenente ambra del Baltico e lapislazzuli dell'Egitto ${ }^{10}$.

Oggi globalizzazione significa un movimento "tettonico" del pianeta di ritorno agli equilibri "naturali" che lo hanno retto fino alla Rivoluzione Industriale: la quota di lavoratori industriali presenti in Asia torna a combaciare con la quota di popolazione mondiale presente nel medesimo continente. Nonostante le illusioni di Trump, quei posti di lavoro non potranno tornare indietro se non in minima parte: quella corrispondente a un innalzamento della qualità produttiva e tecnologica capace di reggere alla concorrenza. Per duecento anni l'Occidente ha alterato in modo artificiale gli equilibri del pianeta costringendo gli altri sei settimi della popolazione mondiale a subire l'egemonia industriale e coloniale del settimo privilegiato. Se la politica non riesce (in fretta) a darsi una inedita dimensione sovranazionale di governo, è giocoforza prevedere che questo sommovimento epocale produca conflitti di ogni tipo. È già successo, del resto. La prima globalizzazione venne drammaticamente arrestata dalla Grande guerra. E fa un certo effetto rileggersi La grande illusione, best-seller uscito nel 1910 e scritto da Norman Angell, futuro premio Nobel nel $1933^{11}$. Sosteneva che la grande illusione fosse proprio la guerra: ormai inutile in un tempo dominato dagli scambi internazionali senza più ruolo per le frontiere degli Stati. Incarnava le speranze di molti ma, come sappiamo, quello stesso titolo venne scelto da Renoir per uno dei primi capolavori della storia del cinema dedicato proprio agli orrori del primo conflitto mondiale.

Sono soltanto spunti confusi che vorrei proporre alla discussione del libro di Marino, in controtendenza a un eccesso di reductio ad unum che trovo nella sua almeno tendenziale identificazione tra globalizzazione e neoliberismo e forse anche a una sua considerazione della natura (ma qui mi spenzolo in un terreno filosofico che proprio non conosco) come troppo immobile nel tempo e, per così dire, "pre-darwiniana", cioè priva di un'evoluzione che si svolge in parallelo all'azione umana, almeno nel corso della breve stagione che agli esseri umani (in confronto a insetti e montagne) è stata offerta di vivere sulla Terra. Ma ringrazio Marino per la mirabile e coraggiosa occasione che ci offre.

\footnotetext{
${ }^{10}$ Cfr. LUCASSEN, Jan, LUCASSEN, Leo, Globalising migration history: the Eurasian experience (16th-21st centuries), Leiden-Boston, Brill, 2014.

${ }^{11}$ ANGELL, Norman, The great illusion: a study of the relation of military power to national advantage, London, William Heinemann, 1912.
} 


\section{L'AUTORE}

Giovanni GOZZINI è professore di Storia contemporanea e Storia del giornalismo alla Facoltà di Lettere e filosofia dell'Università di Siena. È autore di studi su Firenze nell'Ottocento (Firenze francese, Firenze, Ponte alle grazie, 1989; Il segreto dell'elemosina, Firenze, L. S. Olschki, 1994) sulla storia dello sterminio nazista (La strada per Auschwitz, Milano, Bruno Mondadori, 1996), sulla storia del Pci (Storia del Partito comunista italiano, v.7, Dall'attentato a Togliatti all'VIII congresso, Torino, Einaudi, 1998), sulla storia del giornalismo (Storia del giornalismo, Milano, Bruno Mondadori, 2000) sulla storia delle migrazioni internazionali (Le migrazioni di ieri e di oggi. Una storia comparata, Milano, Bruno Mondadori, 2006) e sulla storia della globalizzazione (Un'idea di giustizia, Torino, Bollati Boringhieri, 2010). È membro della direzione della rivista «Passato e presente», dal 2000 al 2007 è stato direttore del Gabinetto Vieusseux di Firenze e dal 2007 al 2008 assessore alla cultura del Comune di Firenze.

URL: < http://www.studistorici.com/progett/autori/\#Gozzini > 